 \\ Clinical Studies and Medical Case Reports
}

\section{A Huge Vaginal Prolapsed Pedunculated Uterine Leiomyoma: A Case Report}

\author{
Incim Bezircioglu', Derya Kilic Sakarya ${ }^{2}$, Mehmet Hakan \\ Yetimalar', Emine Kayhan', Askin Yildiz', Emre Baser ${ }^{1}$ and \\ Burcu Kasap ${ }^{3 *}$
}

${ }^{1}$ Department of Obstetrics and Gynecology, Izmir Katip Celebi University Ataturk Training and Research Hospital, Izmir, Turkey

${ }^{2}$ Department of Obstetrics and Gynecology, Bozkir State Hospital, Konya, Turkey

${ }^{3}$ Department of Obstetrics and Gynecology, Muğla Sıtkı Koçman

University, School of Medicine, Mugla, Turkey

\begin{abstract}
Leiomyomas are the most common benign tumors of the uterus Some of submucousal myomas become pedunculated in the uterine cavity, dilate the cervix and protrude into the vagina. Also the myomas may become necrotic, infected and gangrenous cause of inadequate blood circulation through a long pedicle or if the pedicle of leiomyoma twists. In this report, we present a 46-year old nulliparous woman with a challenging huge vaginal prolapsed pedunculated necrotic, infected $24 \times 14 \times 10 \mathrm{~cm}$ leiomyoma. The patient underwent vaginal myomectomy. On the 6 month follow-up subtotal uterine prolapse regressed, and uterus returned to its normal position. As in the case we present, vaginal myomectomy is a feasible, easy and effective procedure even for the patients with huge leiomyomas.
\end{abstract}

Keywords: Leiomyoma; Myomectomy; Vagina

\section{Introduction}

Leiomyomas are the most common benign tumors of the uterus [1]. They are classified as subserosal, intramural and submucosal on the basis of their location in the uterus. Some of submucousal myomas become pedunculated in the uterine cavity. They dilate the cervix and protrude into the vagina [2]. The patients present with a vaginal mass usually infected and necrotic. There are different approaches on treatment of pedunculated prolapsed myomas, and are a few reports about the management of them in the literature [3].

In this report, we present a patient with a challenging huge, necrotic and infected vaginal prolapsed pedunculated myoma

*Corresponding author: Burcu Kasap, MD, Department of Obstetrics and Gynecology, Muğla Sıtkı Koçman University, School of Medicine, Mugla, 48000, Turkey, Tel: +90 2522114800/5156; E-mail: burcuharmandar@gmail.com; burcuharmandar@mu.edu.tr

Citation: Bezircioglu I, Sakarya DK, Yetimalar MH, Kayhan E, Yildiz A, et al., (2015) A Huge Vaginal Prolapsed Pedunculated Uterine Leiomyoma: A Case Report. J Clin Stud Med Case Rep 2: 005

Received: December 16, 2014; Accepted: January 22, 2015; Published: February 05, 2015 presented with intense acute pelvic pain and severe anemia treated by vaginal myomectomy easily with good prognosis.

\section{Case Report}

A 46-year old schizophrenic nulliparous woman referred with a huge necrotic, infected mass protruding from vagina. The patient was agitated for the intense acute pelvic pain, probably due to the acute necrosis and infection. A reliable history could not be obtained due to her psychosis and as we learned from her relatives, the patient had a history of vaginal bleeding and urinary incontinence for 3 months; however they had just noticed the mass by the acute pain. The urinary incontinence is thought to be related with the huge mass that protruding from the vagina; which pulls down the uterus and vagina and impairs the pelvic normal function.

On gynecological examination, the cervix prolapsed out of the vagina and dilated up to $5 \mathrm{~cm}$ diameters by a pedicle of a huge mass protruding out of the introitus. The dimensions of the mass were $24 \times 14 \times 10 \mathrm{~cm}$ and it was dark brown, necrotic, ulcerated, and infected. It was twisted on its pedicle 3 times (Figure 1). The thigh and vulva was edematous and indurate. On ultrasound examination, uterus and both ovaries appeared normally. The patient had a pulse rate of 128 beats per minute, a blood pressure of $80 / 40 \mathrm{mmHg}$ and her body temperature was $36^{\circ} \mathrm{C}$. The respiratory rate was 16 cycles per minute. Her laboratory tests were challenging asher hemoglobin level was $2.9 \mathrm{gr} / \mathrm{dL}$. Repeat hemoglobin was consistent with the first one. Her hematocrit value was $9.68 \%$, mean corpuscular volume was $50.9 \mathrm{fL}$. Her leukocyte value was $16.700 / \mathrm{mm}^{3}$, platelet value was $719.000 / \mathrm{mm}^{3}$, and INR was 1.29. Renal and hepatic function tests and other requisite biochemical parameters were in normal range. She was in ill-septic state. The patient could not tolerate the examination with Magnetic Resonance Imaging (MRI) or Computerized Tomography due to her agitation and psychosis, but abdominal ultrasonographic examination revealed no pathological finding that can be associated with intraabdominal bleeding or any other disease.

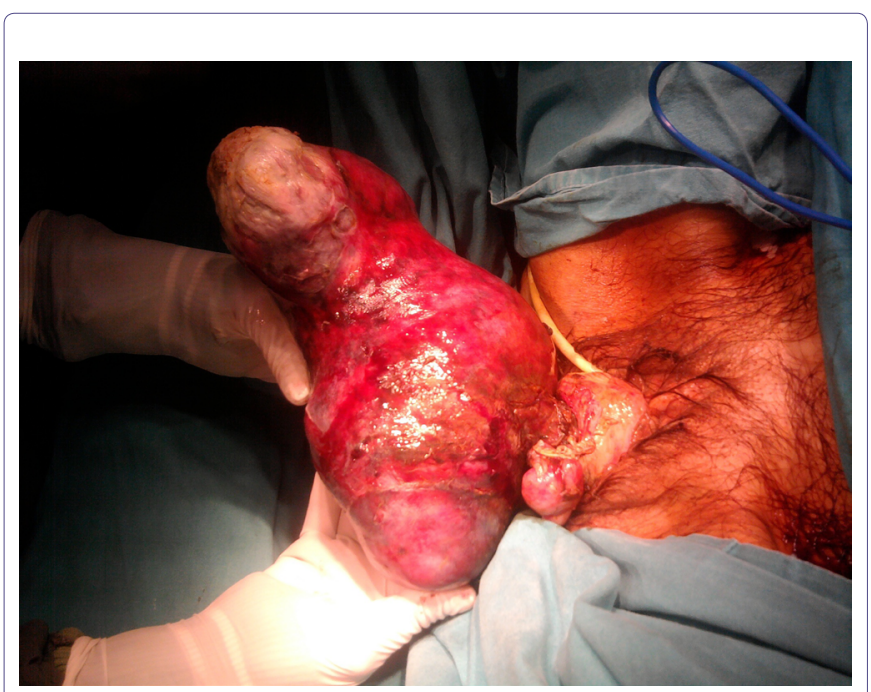

Figure 1: A huge mass protruding out of the introitus. 
Moxifloxacin (800 mg/day intravenous) and Metronidazol (1500 $\mathrm{mg} /$ day intravenous) were administered along with the transfusion of 4 units of Red Blood Cells (RBC) and 4 units of Fresh Frozen Plasma (FFP). Post transfusion hemoglobin level was $5.38 \mathrm{gr} / \mathrm{dl}$ and hematocrit value was $16.8 \%$.

Examination under general anesthesia revealed a huge necrotic, degenerated and ulcerated mass filling the vagina, extending from introitus. The cervix could not be differentiated clinically. The pedicle was $4 \mathrm{~cm}$ diameters in width and whirled round itself 3 times. There was another $3 \times 3 \mathrm{~cm}$ polypoid lesion located on left part of cervix. Transvaginal myomectomy was the approach chosen for the patient.

First we removed the small polypoid lesion with monopolar diathermy. After the distortion of the huge fibroid, we clamped and ligated its pedicle. We excised the mass and replaced the cervix back in to the vagina. The estimated blood loss was $50 \mathrm{ml}$ and the operation took a 10 minutes time. The recovery period was just 2 days.

The excised tumor was $24 \times 14 \times 10 \mathrm{~cm}$ in size and $3 \mathrm{~kg}$ in weight. Pathology confirmed ulcerated and degenerated leiomyoma which contains cystic degeneration and hemorrhagic areas and endocervical polyp.

At the first postoperative day, pelvic examination revealed that the cervix was in normal localization and the edema of surrounding tissues resolved. The patient had a pulse rate of 80 beats per minute, a blood pressure of $90 / 60 \mathrm{mmHg}$ and a body temperature of $36^{\circ} \mathrm{C} .2$ units of additional RBC and 1 units of FFP were administered, which brought hemoglobin levels to $8.3 \mathrm{gr} / \mathrm{dL}$ and hematocrit value to $25 \%$. Her new leukocyte count was $18.600 / \mathrm{mm}^{3}$, and platelet value was $566.000 / \mathrm{mm}^{3}$, INR was 1.1 at the first postoperative day. Psychosis was treated with haloperidol and patient was stabilized.

Two and 6 months after the operation, uterus and cervix were in normal localization and there was no complaint of incontinence and abnormal bleeding.

\section{Discussion}

Uterine leiomyoma is the most common gynecological tumor affecting reproductive age group and one third of all hospital admissions to gynecology services [1]. The common symptoms in patients with submucous pedunculated myomas are summarized in Table 1. When a pedunculated submucosal myoma lengthens to endocervical cavity and dilates it, the myoma prolapses to vagina and owing to its heavy weight; it often drags down the uterus, urinary bladder, vaginal wall and causes cervical elongation in the case we presented. It can lead to uterine prolapse, or even uterine inversion [2]. They impair the pelvic anatomy by pulling down the uterus in relation with the bladder and the rectum. And also the myoma becomes necrotic and infected cause of inadequate blood circulation through a long pedicle. If the pedicle of submucosal leiomyoma twists, infarction results, occasionally red, hemorrhagic, gangrenous degeneration occurs [2]. This is a result of poor circulation of blood through a rapidly growing tumor.

The patient we presented in this case was schizophrenic. It reveals why she sought medical attention so late. The extremely profound anemia was considered to be related with longstanding irregular bleedings. Dysregulation of local growth factors, passive congestion, the increased size, necrosis and ulceration of the endometrial surface have been implicated in the irregular episodes of vaginal hemorrhage of the patients with leiomyoma [4].

\begin{tabular}{|l|}
\hline Abnormal bleeding \\
\hline Anemia \\
\hline Discharge (thin, bloody, foul) \\
\hline Lower abdominal pain (acute-chronic) \\
\hline Pressure and heaviness in the pelvis \\
\hline Difficulty with urination or urinary incontinence \\
\hline Constipation \\
\hline Reproductive disfunction, infertility \\
\hline
\end{tabular}

Table 1: The common symptoms in patients with submucous pedunculated myomas.

Vaginal myomectomy is a short, easy and effective procedure; therefore we preferred to perform this technique instead of an extensive surgery after the satisfactory preoperative preparation including correction of anemia. Imaging modalities especially ultrasonography and MRI play an important role in the management of patients with prolapsed pedinculated leiomyomas especially for the pre-operative localization of leiomyomas and surgical planning for myomectomy. Most authors regard this surgical procedure as the safe and initial treatment for prolapsed pedunculated submucous myomas $[5,6]$. It reduces the operating time, anesthetic exposure and intraoperative bleeding. Golan et al., reported in their series that the technique was successful in $95.6 \%$ of the patients and all had uneventful recoveries [5]. The associated morbidity was minimal and the recovery period was very short as the case we presented [5].

In well-selected patients even for huge leiomyomas, myomectomy can be performed by vaginal route with no skin incision. With further review, advantages of this technique may be demonstrated.

\section{Conclusion}

In this case we have presented a huge prolapsed pedinculated submucosal myoma in a schizophrenic woman and the treatment results by vaginal myomectomy procedure. Vaginal myomectomy is an easy, safe and effective procedure which also conservative and fertility preservative. We recommend resection of such tumors as the best possible management by vaginal myomectomy.

\section{Informed Consent}

The patient gave informed consent.

\section{References}

1. Levy BS (2008) Modern management of uterine fibroids. Acta Obstet Gynecol Scand 87: 812-823.

2. Thompson JD, Rock JA (2003) Leiomyomata uteri and myomectomy. In: Te Linde's text book operative gynecology,(9th edn). Lippincott-Raven, New York, USA. pp 256-261.

3. Faivre E, Surroca MM, Deffieux X, Pages F, Gervaise A, et al. (2010) Vaginal myomectomy: literature review. J Minim Invasive Gynecol 17: 154-160.

4. Wegienka G, Baird DD, Hertz-Picciotto I, Harlow SD, Steege JF, et al. (2003) Self-reported heavy bleeding associated with uterine leiomyomata. Obstet Gynecol 101: 431-437.

5. Golan A, Zachalka N, Lurie S, Sagiv R, Glezerman M (2005) Vaginal removal of prolapsed pedunculated submucous myoma: a short, simple, and definitive procedure with minimal morbidity. Arch Gynecol Obstet 271: 11-13.

6. Goldrath MH (1990) Vaginal removal of the pedunculated submucous myoma: historical observation and development of new procedure. J Reprod Med 35: 921-924 